Table 2 | Probability of exact allelic matching at 16 microsatellite loci in a population of Afghan hounds

\begin{tabular}{ll}
\hline Scenario & Probability \\
\hline Hardy-Weinberg* & $7 \times 10^{-4}$ \\
Full siblings $\dagger$ & $9 \times 10^{-6}$ \\
Inbred $F=0.25 \ddagger$ & $3 \times 10^{-8}$ \\
Pedigrees & $4 \times 10^{-4}$ \\
\hline
\end{tabular}

A random sampling of the population, assuming Hardy-Weinberg conditions.

$\uparrow$ Assuming the clone is a full sibling of the donor.

$\ddagger$ Assuming everyane in the population is related with an inbreeding coefficient of 0.25 .

s.An assumed pedigree in which the donor is the product of a mother-san mating, and the putative clone is the product of

crossing the danor back to the mother.

The probability that the putative clone should have precisely the same genotype as the donor was computed for different assumptions regarding the relatedness of the sample and the donor ${ }^{7}$ (Table 2). In all cases, the allele frequencies were computed from a sample of 11 AKC-registered Afghan hounds plus the donor. According to genetic maps of the canine chromosomes, the 14 mapped markers were unlinked to one another, so each microsatellite was treated as an independent locus 89 .

The match probabilities ranged from $7 \times 10^{-14}$ for unrelated dogs to $4 \times 10^{-4}$ for those with a specific inbred pedigree. A higher degree of inbreeding would increase the match probability further, but the donor does not seem to be extremely inbred; both the donor and Snuppy are heterozygous at 8 of the 16 markers, which is not significantly different from the number of heterozygous markers expected, given the observed allele frequencies and no inbreeding.

Mitochondrial D-loop sequencing revealed 26 variable bases within the 614 analysed (Table 3). Snuppy and the donor dog differed at 12 of the 26 sites. Nine of the Afghan hound sequences disagreed with each other at only one base (position 548) and differed from the donor by only three to four bases. Also, the two Labrador retrievers had identical mitochondrial sequences that differed from the donor by only three bases. The sequence from Snuppy differed from that of any other dog at 9-14 sites.

These data are consistent with Snuppy being a genetic clone of the donor dog Tai. Our analysis rules out most feasible alternatives to a true clone, such as the production of a delayed twin, which would have produced dogs with the same mitochondrial D-loop sequence, or an animal resulting from extreme inbreeding, which would have yielded dogs

Table 3| Mitochondrial genotypes at 26 polymorphic loci within the canine mitochondrial D-loop

Locit $\quad 4976$ no nB B5 1431481501531551591621661753043233343383743547848252654856562

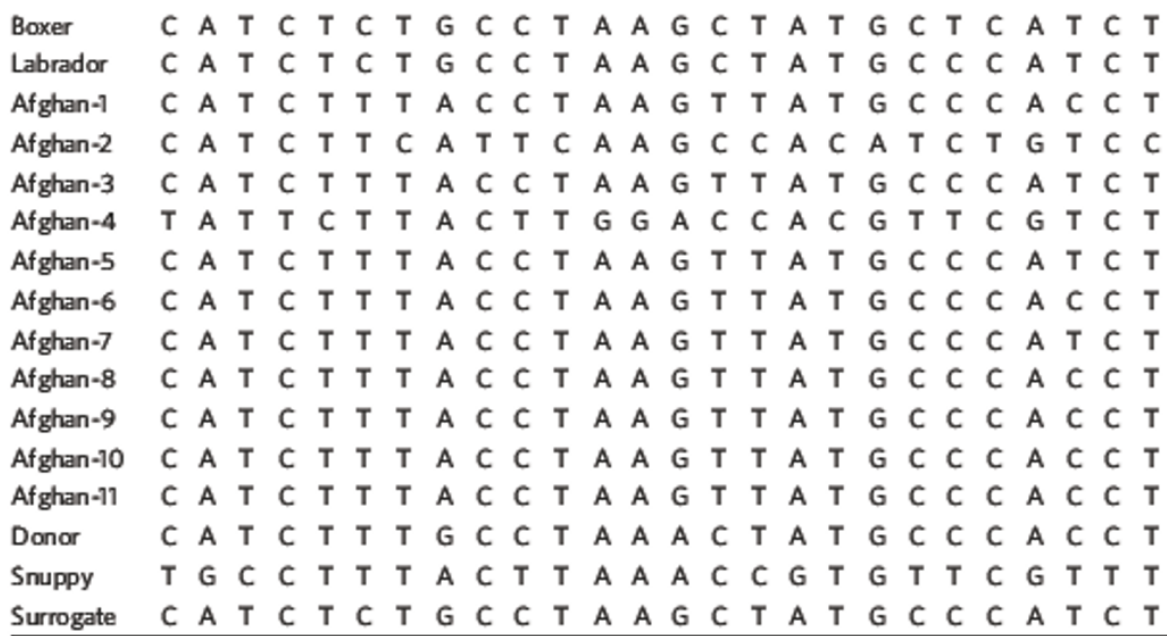

"The locations of the variable bases are given relative to the 5 ' end of the forward primer used to amplify and sequence the region.

The entire length of the segment is 614 bases. that were homozygous at more than the observed eight loci.

Conclusions drawn from these results are subject to caveats. First, we did not witness the drawing of the blood samples, which was done under the supervision of a third party. However, no obvious hypothetical manipulation of the samples would have generated the results described here - perfect matching of the nuclear markers, and distinct differences between the mitochondrial sequences for the donor and Snuppy. Second, we were not provided with samples from the oocyte donor, although tissue samples from this dog have been tested by investigators at Seoul National University ${ }^{10}$. Without this sample, we are unable to confirm the original experimental details ${ }^{1}$, or to say with certainty that the mitochondrial variants observed were those that were expected.

Finally, our statistical analysis is based on a limited number of Afghan hounds. A larger number of unrelated individuals might have provided a more precise estimate of population allele frequencies. However, given that the dogs tested are representative of this relatively restricted breed and that many share a partial heritage with the donor, the statistical conclusions are conservative.

Heidi G. Parker*, Leonid Kruglyakt,

Elaine A. Ostrander*

tCancer Genetics Branch, National Human

Genome Research Institute, National Institutes of Health, Bethesda, Maryland 20892, USA

e-mait: eostrand@mail.nih.gov

†Lewis-Sigler Institute for Integrative Genomics, and the Department of Ecology and Evolutionary Biology, Carl Icahn Laboratory, Princeton

University, Princeton, New Jersey 08544, USA

1. Lee, B.C. etal. Nature 436,641(2005)

2. Hwang WS et al. Sclence 303,1669-1674(2004).

3 Hweng W. S eta. Sclence 308, 1777-1783(2005).

4. Lindblad-Toh, K et al Nature 438, 803-819 (2005)

5. DeNise, S etal. Anim. Genet. 35, 14-17 (2004).

6. Halverson, I\& Basten C.J. Foren. Sd. 50, 352-363 (2005).

7. Weir B.S. Genetic Data Analysis II (Sinauer, Sunderland Massachusetts, 1996).

8. Mellersh C.S.et al. Mammal. Gename 11,120-130 (2000)

9. Guyon, Ret al. Proc Natl Acod. Sci. USA 100,5296-5301 (2003)

10. Secul National University Investigative Committee Nature 440, dok101038/hature04686(2006).

doi:10.1038/nature04685

\section{MOLECULAR GENETICS}

\section{Verification that Snuppy is a clone}

\section{Arising from: B. C. Lee et al. Nature 436, 641 (2005)}

Somatic-cell nuclear-transfer technology has been used to clone a variety of animal species ${ }^{1-3}$, but the overall efficiency of the cloning process and the viability of embryos has remained low". Until Lee et al. described the doning of two Afghan hounds by nudear transfer from adult skin fibroblasts into oocytes that had matured in vivo ${ }^{5}$, dog cloning had been unsuccessful because of the difficulty of collecting canine oocytes matured in vivo at metaphase II (ref. 6). Here we provide independent evidence from the Seoul National University Investigation Committee that Snuppy, the one of the pair to survive, is a genuine done.
To investigate whether the cloned dog was genetically identical to the donor Afghan, we obtained blood samples from Snuppy, from the male Afghan hound that provided the somatic cell, and from the surrogate mother. In addition, autopsy samples from the sincedeceased mixed-breed dog that originally provided the egg used to create Snuppy were obtained from the research team who generated the cloned dog. DNA was extracted from the blood and autopsy samples and used for microsatellite analysis of genomic DNA and nucleotide sequences of mitochondrial DNA. 
Table1| Analysis of canine-specificmicrosatellite loci

\begin{tabular}{|c|c|c|c|c|c|c|c|c|}
\hline \multirow[t]{2}{*}{ Canine markers ${ }^{*}$} & \multicolumn{2}{|c|}{$\begin{array}{l}\text { Snuppy } \\
\text { (blood leukocytes) }\end{array}$} & \multicolumn{2}{|c|}{$\begin{array}{l}\text { Donor Afghan } \\
\text { (blood leukocytes) }\end{array}$} & \multicolumn{2}{|c|}{$\begin{array}{l}\text { Surrogate } \\
\text { (blood leukocytes) }\end{array}$} & \multicolumn{2}{|c|}{$\begin{array}{l}\text { Egg donor" } \\
\text { (sutopsy lung tissue) }\end{array}$} \\
\hline & Peak 1 & Peak 2 & Peak 1 & Peak 2 & Peak 1 & Peak 2 & Peak1 & Peak 2 \\
\hline AHTा11 & 72 & 78 & 72 & 78 & 78 & 80 & 76 & 80 \\
\hline AHT137 & 130 & 148 & 130 & 148 & BO & 148 & 130 & 142 \\
\hline$C 01.424$ & 186 & १८८ & 186 & 188 & १८६ & 188 & 182 & 188 \\
\hline Co7.620 & 106 & $n_{4}$ & 106 & 114 & $n 2$ & 114 & 114 & 124 \\
\hline Wilms-TF & 291 & & 291 & & 291 & & 297 & 299 \\
\hline $\mathrm{FH} 2274$ & 278 & 286 & 278 & 286 & 278 & & 278 & 282 \\
\hline $\mathrm{FH} 2289$ & 284 & 328 & 284 & 328 & 296 & & 288 & 292 \\
\hline REN210K18 & 216 & & 216 & & 212 & 217 & 202 & 217 \\
\hline
\end{tabular}

"Eight of 27 canine-specific markers used for microsatellite assay are shown.

Table 2 |Sequence aligmments within D-loop region, cytochrome $b$, and 16Sr RNA of mtDNA

\begin{tabular}{|c|c|c|c|c|c|c|c|c|c|c|c|c|}
\hline & \multicolumn{12}{|c|}{ Nuc leotide positions } \\
\hline & \multicolumn{9}{|c|}{ D-loap region (409 bp) } & \multirow{2}{*}{$\begin{array}{c}\text { Cytochrome b (380 bp) } \\
426\end{array}$} & \multicolumn{2}{|c|}{165 rRNA (440 bp) } \\
\hline & 170 & 175 & 195 & 343 & 354 & 455 & 498 & 546 & 568 & & 1117 & $\mathrm{n45}$ \\
\hline Snuppy & A & $\mathbf{T}$ & A & C & G & $\mathbf{T}$ & $\mathbf{T}$ & G & $\mathbf{T}$ & G & C & G \\
\hline Donor Afghan & G & C & A & $\mathbf{T}$ & A & C & C & A & C & A & $\mathbf{T}$ & A \\
\hline Surrogate & G & C & G & $\mathbf{T}$ & A & C & C & A & $\mathbf{T}$ & A & C & A \\
\hline Egg donor & A & $\mathbf{T}$ & A & C & G & $\mathbf{T}$ & $\mathbf{T}$ & G & $\mathbf{T}$ & G & C & G \\
\hline
\end{tabular}

"The nucleotide positions were counted from the start, the initistion codon, and the transcriptional start of the D-loop, cytochrame b, and 16S rRNA sequences, respectively (Gen Bank accession number AY729880). bp, Base pairs.

The microsatellite analyses were performed with genomic DNA from four dogs (Snuppy, the donor Afghan, the surrogate mother and the egg donor) using 27 canine-specific markers that allow extremely inbred animals to be distinguished. As shown in Table 1, which shows only 8 of 27 loci used for microsatellite analysis, Snuppy and the donor Afghan have identical microsatellite patterns for all loci, whereas the surrogate mother evidently has a different genetic origin.

For 18 of the microsatellite loci analysed (including those shown in Table 1), the egg donor and Snuppy showed distinct microsatellite patterns. The microsatellite loci used for the analyses show enough variation among Afghan hound individuals to allow them to be distinguished (results not shown). Thus, the Committee's microsatellite analysis of genomic
DNA demonstrates that the cloned dog Snuppy is genetically identical to its fibroblastdonor Afghan hound.

If Snuppy is a cloned dog, his mitochondrial DNA (mtDNA) should be identical to that of the egg donor, but not to that of the fibroblast-donor Afghan. To test this, we determined partial sequences of four regions of the dog mtDNA. Based on the complete nucleotide sequence (GenBank accession number U96639), four primer sets were synthesized and used for polymerase chain reaction $(\mathrm{PCR})^{7}$ : the cytochrome $b$ gene (L14,252-L14,631), the $16 \mathrm{~S}$ ribosomal RNA gene (L2,033-L2,472), and the two overlapping hypervariable $\mathrm{D}$-loop regions (L15,622-L16,030 and L15,485-L16,090). PCR products were sequenced and a BLAST search confirmed their identity as mtDNA.

The sequence alignments of 409 base pairs of the hypervariable D-loop region revealed eight mismatches between the mtDNA of Snuppy and the donor Afghan (Table 2). A non-match was observed even in more conserved regions (one mismatch in the cytochrome $b$ gene and two mismatches in the $16 \mathrm{~S}$ rRNA gene). These results indicate that Snuppy could not have been produced by splitting the early blastomere from which the donor Afghan originated. The sequence alignments also revealed a perfect match between Snuppy and the egg donor, and a non-match between Snuppy and the surrogate mother (Table 2). This is consistent with Snuppy being a nuclear clone of the donor Afghan.

On the basis of the results of both a microsatellite analysis of genomic DNA and a sequence comparison of mtDNA, it is highly unlikely that Snuppy came either from extreme inbreeding or from blastomere separation (also known as twinning). It is virtually certain that Snuppy was generated from somatic-cell nuclear transfer, as claimed by Lee et $a l^{5}$.

Seoul National University Investigation Committee ${ }^{\star}$, Jung B in Leeł, Chankyu Park Office of Research Affairs, Seoul National University, Gwanak-gu, Seoul 151-742, South Korea e-mait: mhchung@snuac.kr tDepartment of Forensic Medicine, Seoul National University College of Medicine, 28 Yeongon-dong, Chongno-gu, Seoul 110-799, South Korea †Department of Life Sciences, Korea Advanced Institute of Science and Technology, Yusong-gu, Taejon 305-701, South Korea

1. Wilmut, Let al. Nature 385, 810-813 (1997).

2. Wakayama, T.etal. Nature394, 369-374(1998).

3. Zhou Q et al Science 302, 1179 (2003).

4. Sutowsky, P. \& Prather, R. S Trends Biotech 22, 205-208 (2005).

5. Lee, B.C. et a. Nature 436, 641 (2005).

6. Westhusin, M. E.etal.J. Reprod Fert. Suppl. 57, 287-293 (2001).

7. Kim, K. S.et a. Genes Genet Syst. 76, 243-250 (2001).

doi:101038/nature04686 